\title{
Cure and prevention of cardiovascular diseases: herbs for heart
}

\author{
Suman Ray ${ }^{*}$ iD and Mahesh Kumar Saini
}

\begin{abstract}
Herbs contribute to more than 60-70\% in development of modern medicines in the world market either directly or indirectly. The herbal treatments for congestive heart failure, systolic hypertension, angina, atherosclerosis, cerebral insufficiency and venous insufficiency etc. has been known since ancient times. Unlike allopathic medicines, Ayurveda medicines are considered safe, however, the adverse reactions of herbal drugs is also reported. In this paper, we have compiled 128 herbs and their parts that have medicinal value to prevent, alleviate or cure heart disease related disorders. Jaccard Neighbour-joining cluster analysis using Free Tree software was used to assess the relative importance of plants in context with its healing potential for heart related disease. Based on the medicinal value in context with the heart, five major clusters of the selected 128 herbs were made. Correlation of the distance between herbs revealed that most of these herbs were found to have more than one medicinal property. The distance in dendogram depicted closeness of properties curing heart disease; as less the distance between two medicinal plants or two groups they will more close to cure particular heart disease. During drug development, a medicinal plant can be replaced by another plant of same group or by another plant of its neighbour group but from same pedigree. Thus, in case of non-availability of herbs or if it belongs to the category of rare, threatened, and endangered species, such method may add to new ways of drug development.
\end{abstract}

Keywords: Healthcare, Herbs, Cardiovascular diseases, Hypertension, Herbal drugs

\section{Introduction}

According to WHO, cardiovascular diseases (CVDs) kill 17.9 million people per year, accounting for $31 \%$ of all global deaths. Heart attacks and strokes account for $80 \%$ of CVD deaths [1]. Populations in low and middle income countries (LMICs) contribute $75 \%$ of the CVD deaths [2]. It is predicted that by 2030 more than 22.2 million people will die annually from CVDs. Due to high prevalence of CVD among older adults in LMIC, population with CVD has increased and become a major challenge in future for the health care system. The therapeutic potential of herbs in healthcare system is well known in all over the world whether it is for diseased state or proper maintenance of health [3, 4]. Since Ayurvedic medicines belong to natural sources, they are

\footnotetext{
* Correspondence: suman@nistads.res.in; sumanitrc@gmail.com CSIR-National Institute of Science Technology and Development Studies (CSIR-NISTADS), Pusa Gate, K.S. Krishnan Marg, New Delhi 110 012, India
}

considered safe compared with allopathic medicines. However, many adverse reactions of herbal drugs is also reported [5, 6]. Ayurvedic medicines can cause adverse effect if the patients continue to take medicines with no monitoring. Prolonged use or overdose of herbal medications lead to side effects e.g. high risk of cardiovascular events. A major drawback is the lack of information on the social and economic benefits on the industrial utilization of medicinal plants $[7,8]$. The standard pharmacovigilance techniques (WHO guidelines) when applied presents challenges such as the ways in which herbal medicines are regulated, used, named, and perceived [9]. Very often patient undergo medication with Allopathic and Ayurvedic medicines simultaneously and dose-related responses are rarely measured and reported. Conventional pharmacovigilance tools, such as prescription-event monitoring and the use of computerized health record databases, for evaluating the safety of herbal medicines has limitations too. Reporting of 
adverse events possibly caused by herbal and traditional medicines in an extensive manner is needed for the systematic and rational use of drugs [9].

Since herbal medications do not require proof of efficacy and safety and there is lack of enough clinical data on herbal medication although, most of the herbs demonstrate an effect on biological mechanisms [10]. Clinical studies so far on herbal medications are limited in sample size and its impact on relevant clinical outcomes is not much studied. There is increased risk of side effects as it is not tested in pregnant women and children. Sometimes there is even contamination with other conventional medications and there also exists risk of drug interactions. Even substitution with alternative plant species is also reported. Enough and improved knowledge herbal medications is essential. Also, there should be transparency between patient- physician and possible benefits, side effects should be discussed. Thus, herbal drug development is possible only if there is development of standardized herbal products.

The present study was conducted to assess the relative importance of herbs that has medicinal potential to regulate heart and cure related disease. This may add to enhance drug discovery approaches for its promotion and development i.e. to generate safety data-either before or after marketing of the formulation.

\section{Materials and methods}

Plants having medicinal properties for treatment of various heart diseases were extracted from secondary database search e.g. Google Scholar, PubMed and published research articles. Based on this, dataset of 128 different medicinal plants were further grouped and assessed for its relative medicinal potential to regulate and treat heart disease (Table 1 [12-27] http://www.nmpb.nic.in/, https://en.wikipedia.org/wiki/Scutellaria\#Traditional_ use). The percentage of plant parts used for the treatment of heart disease were further assessed (Fig. 1). Heart disease and the Plant uses were categorised in three parts: Plants used in only one diseases of heart, two disease of heart, three diseases of heart were grouped together (Table 2 [27]). Jaccard Neighbourjoining dendrogram were obtained through collected dataset of 128 different plants useful for heart disease ailment, computed performed with the help of Free Tree software version 0.9.1.50 and FigTree version 1.2.2. And mathematical consensus tree so obtained after 1000 replicates of bootstrap. Herbs were recorded as ' 1 ' for present or ' 0 ' for absent of a particular medicinal property related to heart. The objective of the cluster analysis was to develop sub grouping of plants on the basis of their properties to treat heart disease. This method of clustering not only clusters sample, but also it clusters various clusters that were formed earlier in the clustering process. In this method, each sample or variable was treated as a cluster of 1 and the closest two clusters are joined to form a new cluster [28].

\section{Results}

In this paper we have listed 128 plants, its habit and parts used for treatment of heart disease (Table 1). Comparative analysis of parts used of percentage of total plants showed that root and rhizome is the most frequently used plant parts followed by leaves, while gum being the least frequently used plant parts in context with ailment of heart disease (Fig. 1). The order (maximum to minimum) of plant parts used for heart disease ailment is root and rhizome-leaf-stem-flower-fruit and seed-other parts-gum (Fig. 1). Analysis of plants used for various types of heart diseases showed that only one plant i.e. Crocus sativus $L$. has the potential to cure five types of heart disease-hypertension, heart attack and reduction in blood fat, anti-oxidant and cardiac tonic thus indicating its relevancy in context with cardiovascular diseases (Table 2). Similarly, plants having medicinal property to cure four heart disease category were also few (Table 2). Maximum number of plants were found to be in category of curing only one heart disease type. Very few plants were observed to have medicinal property capable of treating multiple heart disease type (Table 2).

Cluster analysis based on Jaccard Neighbour-joining dendrogram using collected dataset of 128 herbs useful for heart with the help of Free Tree software version 0.9.1.50 and FigTree version 1.2.2. and mathematical consensus tree was obtained after 1000 replicates of bootstrap (Fig. 2). Five major clusters of the selected 128 herbs were observed on the basis of their medicinal value in context with heart. Within a cluster most of herbs showing similar properties and medicinal similarity negatively correlated with the cluster distance. Correlation of the distance between herbs also revealed that most of these herbs were found to have more than one medicinal property (Fig. 2).

\section{Discussion}

A variety of modern medicines have been developed from herbs that are being used by native people [29]. Herb serve as both preventive and therapeutic purposes of many diseases. Use of herbs for cardiovascular diseases such as congestive heart failure, systolic hypertension, angina pectoris, atherosclerosis, cerebral insufficiency, and arryhythmia is prevalent since ancient time [30]. Herbs has been a continuing source for medicine e.g. antineoplastic drug paclitaxel derived from Taxus brevifolia, digitoxin from Digitalis purpurea, reserpine from Rauwolfia serpentina etc [1]. These herbs are used for treatment of cardiovascular diseases. Use of 
Table 1 List of plants, its habit and parts used for treatment of heart disease

\begin{tabular}{|c|c|c|c|c|c|}
\hline $\begin{array}{l}\text { S. } \\
\text { No. }\end{array}$ & Scientific name & Common name & Family name & Habit & Part used \\
\hline 1 & Achillea millefolium & Common Yarrow & Asteraceae & Herb & Fruit (Seed) \\
\hline 2 & Acorus calamus & Calamus & Acoraceae & Herb & Root (Rhizome) \\
\hline 3 & Actaea racemosa & Black cohosh & Ranunculaceae & Herb & Leaf and stem (http://www.nmpb.nic.in/) \\
\hline 4 & Allium sativum & Garlic & Amaryllidaceae & Herb & Root \\
\hline 5 & Aloe vera & Aloe vera & Asphodelaceae & Herb & Leaf \\
\hline 6 & Amomum subulatum Roxb & Black cardamom & Zingiberaceae & Herb & Leaf, Seed, Fruit \\
\hline 7 & Anchusa italica Retz. & Anchusa & Boraginaceae & Herb & Flowers \\
\hline 8 & Anethum graveolens & Dill & Apiaceae & Herb & Fruit (Seed), Fruit \\
\hline 9 & Anthemis gayana Boiss. & Mayweed & Asteraceae & Herb & Leaf and flower \\
\hline 10 & Apium graveolens & Ajmoda & Apiaceae & Herb & Fruit \\
\hline 11 & Aquilaria agallocha Roxb. & Agarwood & Thymelaeaceae & Tree & Stem Wood \\
\hline 12 & Arctium minus hill. & Lesser burdock & Compositae & Herb & Root \\
\hline 13 & Bambusa arundinacea Retz. & Bans & Poaceae & Shrub & Leaf \\
\hline 14 & Berberis darwinii & Barbery & Berberidaceae & Herb & Rhizomes [11] \\
\hline 15 & Berberis integerrima & Zerešk & Berberidaceae & Shrub & Fruit \\
\hline 16 & Berberis vulgaris & Common barberry & Berberidaceae & Shrub & Fruit \\
\hline 17 & Boerhavia diffusa & Puarnava & Nyctaginaceae & Herb & Root, Whole Plant \\
\hline 18 & Boswellia sacra & Olibanum-tree & Burseraceae & Tree & Gum resin [12] \\
\hline 19 & Calamintha acinos (L.) Clairv. & calamints & Lamiaceae & Herb & Aerial parts \\
\hline 20 & Calendula officinalis $L$. & English marigold & Asteraceae & Herb & Flowers \\
\hline 21 & Camellia sinensis & Tea & Theaceae & Shrub & Leaf \\
\hline 22 & Capsicum annuum & Capsicum & Solanaceae & Herb & Fruit \\
\hline 23 & Carissa carandas linn. & Karanda & Apocynaceae & Shrub & Fruit \\
\hline 24 & Carthamus tinctorius & Safflower & Asteraceae & Herb & Fruit (Seed) \\
\hline 25 & Centaurea behen $L$. & Behen & Compositae & Herb & Roots \\
\hline 26 & Centella asiatica (I.) Urban & Gotu kola & Apiaceae & Herb & Leaf, Whole Plant \\
\hline 27 & Cichorium intybus & Chicory & Asteraceae & Shrub & Flower, Leaf, Root, Fruit (Seed) \\
\hline 28 & Cinnamomum camphora (L.) & Camphor tree & Lauraceae & Tree & Camphor \\
\hline 29 & Cinnamomum cassia (L.) & Chinese cinnamon & Lauraceae & Herb & Bark \\
\hline 30 & Cinnamomum verum & Cinnamon & Lauraceae & Tree & Bark (Stem), Leaf \\
\hline 31 & Citrus medica L. & Citron & Rutaceae & Tree & Fruit \\
\hline 32 & Commiphora myrrha & Myrrh & Burseraceae & Tree & Oleo-Gum Resin \\
\hline 33 & Commiphora wightii & Gugglul & Burseraceae & Tree & Oleo-Gum Resin, Stem, Root \\
\hline 34 & Coriandrum sativum & Coriander & Apiaceae & Herb & Fruit, Leaf \\
\hline 35 & Crataegus monogyna & Hawthorn & Rosaceae & Tree & Flower and Leaf \\
\hline 36 & Crataegus pontica c. Koch. & Hawberry & Rosaceae & Tree & Fruit \\
\hline 37 & Crocus haussknechtii & Autumn crocus & Iridaceae & Herb & whole plants [13] \\
\hline 38 & Crocus sativus $L$. & Saffron & Iridaceae & Herb & Flowers \\
\hline 39 & Curcuma longa & Turmeric & Zingiberaceae & Herb & Root (Rhizome, Tuber) \\
\hline 40 & Curcuma zedoaria (Bergius) & Zedoary & Zingiberaceae & Herb & Rhizome \\
\hline 41 & Ecbalium elaterium & Squirting cucumber & Cucurbitaceae & Herb & Fruit \\
\hline 42 & Eclipta prostrata & Bhringraj & Asteraceae & Herb & Whole Plant \\
\hline 43 & Elettaria cardamom & Cardamom & Zingiberaceae & Herb & Fruit (Fruit, Seed) \\
\hline
\end{tabular}


Table 1 List of plants, its habit and parts used for treatment of heart disease (Continued)

\begin{tabular}{|c|c|c|c|c|c|}
\hline $\begin{array}{l}\text { S. } \\
\text { No. }\end{array}$ & Scientific name & Common name & Family name & Habit & Part used \\
\hline 44 & Falcaria vulgaris & Sickleweed & Apiaceae & Herb & Leaf, flower and Stem \\
\hline 45 & Fragaria vesca $L$. & Alpine strawberry & Rosaceae & Herb & Leaf, rhizome and Fruit \\
\hline 46 & Garcina indica choisy & Amsul & Clusiaceae & Tree & Fruit (Fruit, Peel) \\
\hline 47 & $\begin{array}{l}\text { Garcinia cambogia (gaertn.) } \\
\text { Desr. }\end{array}$ & Citrin & Clusiaceae & Tree & Leaves and fruits [14] \\
\hline 48 & Garcinia pedunculata roxb. & Amlavettas & Clusiaceae & Tree & Fruit \\
\hline 49 & Genus panax & Ginseng & Araliaceae & Shrub & Root [15] \\
\hline 50 & Ginkgo biloba & Ginkgo & Ginkgoaceae & Tree & Leaves [16] \\
\hline 51 & Gmelina asiatica linn. & Badhar & Lamiaceae & Tree & Bark (Root) \\
\hline 52 & Gundelia tournefortii & Gundelia & Asteraceae & Herb & Leaf \\
\hline 53 & Inula helenium & Elecampane & Asteraceae & Shrub & Whole plant [17] \\
\hline 54 & Juniperus communis & Common juniper & Cupressaceae & Tree & Fruit \\
\hline 55 & Lactuca sativa & Garden lettuce & Asteraceae & Herb & Leaf \\
\hline 56 & Lavandula stoechas $L$. & French lavender & Lamiaceae & Herb & Aerial parts and flowers \\
\hline 57 & Leonurus cardiac & Motherwort & Lamiaceae & Herb & Whole plant [18] \\
\hline 58 & Magnifera indica linn. & Aam & Anacardiaceae & Tree & Fruit \\
\hline 59 & Malus domestica Baumg. & Table apple & Rosaceae & Tree & Peels and fruits \\
\hline 60 & Malva neglecta & Common mallow & Malvaceae & Herb & Leaf and Stem \\
\hline 61 & Medicago sativa & Alfalfa leaves & Fabaceae & Herb & Leaf \\
\hline 62 & Melilotus indicus & Sweet clover & Fabaceae & Herb & Leaf \\
\hline 63 & Melissa officinalis L. & Lemon Balm & Lamiaceae & Herb & Aerial parts and leaf \\
\hline 64 & Mentha $\times$ piperita $L$. & Peppermint & Lamiaceae & Herb & Leaf \\
\hline 65 & Mentha spicata & Spearmint & Lamiaceae & Herb & Leaf \\
\hline 66 & Myristica fragrans & Nutmeg & Myristicaceae & Tree & Fruit \\
\hline 67 & Nardostachys jatamansi & Jatamamsi & Caprifoliaceae & Herb & Root (Rhizome) \\
\hline 68 & Nectaroscordum tripedale & Avon Bulbs & Amaryllidaceae & Herb & Flowers [19] \\
\hline 70 & Nerium oleander $L$. & Nerium & Apocynaceae & Shrub & Leaf and flower \\
\hline 71 & Nigella sativa & Black-caraway & Ranunculaceae & Herb & Fruit (Seed) \\
\hline 72 & Nymphaea alba L. & White nenuphar & Nymphaeaceae & Herb & Flowers \\
\hline 73 & Ocimum bacilicum & Basil & Lamiaceae & Herb & Leaf, Whole Plant, Root, Fruit (Seed) \\
\hline 74 & Olea europaea & Olive & Oleaceae & Tree & Seeds \\
\hline 75 & Paeonia officinalis $L$. & Common peony & Paeoniaceae & Herb & Roots \\
\hline 76 & Paliurus spina-christi miller. & $\begin{array}{l}\text { Christ's thorn } \\
\text { jujube }\end{array}$ & Rhamnaceae & Tree & Fruit \\
\hline 77 & Phyllanthus emblica $L$. & Amla & Phyllanthaceae & Tree & Fruits and Leaves, branches, barks \\
\hline 78 & Picrorrhiza kurroa & Katuka & Plantaginaceae & Herb & Root \\
\hline 79 & Piper longum & $\begin{array}{l}\text { Long pepper } \\
\text { (pippali) }\end{array}$ & Piperaceae & Herb & Root, Fruit \\
\hline 80 & Piper nigrum & Black pepper & Piperaceae & Herb & Stem, Fruit \\
\hline 81 & Pistacia vera $L$. & Pistachio & Anacardiaceae & Tree & Seed [20] \\
\hline 82 & Polypodium vulgare $L$. & Common polypody & Polypodiaceae & Herb & Roots \\
\hline 83 & Portulaca oleracea & Common purslane & Portulacaceae & Herb & Fruit (Seed), Whole Plant \\
\hline 84 & $\begin{array}{l}\text { Prunus scoparia (spach) } \\
\text { schneider }\end{array}$ & Wild almond & Rosaceae & Shrub & Seed [21] \\
\hline 85 & Pterocarpus santalinus L.f. & Red sandalwood & Fabaceae & Tree & Bark and wood \\
\hline
\end{tabular}


Table 1 List of plants, its habit and parts used for treatment of heart disease (Continued)

\begin{tabular}{|c|c|c|c|c|c|}
\hline $\begin{array}{l}\text { S. } \\
\text { No. }\end{array}$ & Scientific name & Common name & Family name & Habit & Part used \\
\hline 86 & Punica granatum linn & Anardana & Lythraceae & Shrub & Flower, Fruit (Fruit, Seed) \\
\hline 87 & Pyrus communis $L$. & European pear & Rosaceae & Tree & leaves and bark and fruit [22] \\
\hline 88 & Rheum ribes & $\begin{array}{l}\text { Rhubarb of } \\
\text { babilonia }\end{array}$ & Polygonaceae & Herb & Stem \\
\hline 89 & Rhus coriaria & Sicilian sumac & Anacardiaceae & Shrub & Leaf and Fruit \\
\hline 90 & Rosa canina & Dog rose & Rosaceae & Shrub & Flowers \\
\hline 91 & Rosa damascena Mill. & Damask rose & Rosaceae & Shrub & Flowers \\
\hline 92 & Rubus caesius & European dewberry & Rosaceae & Shrub & Fruit and Leaf \\
\hline 93 & Rumex crispus & Curly dock & Polygonaceae & Herb & Fruit and leaf \\
\hline 94 & Ruscus aculeatus & Butcher's broom & Asparagaceae & Shrub & Whole plant [23] \\
\hline 96 & Santalum album $L$. & Indian sandalwood & Santalaceae & Tree & Bark and wood \\
\hline 97 & Scutellaria pekinensis & Skullcap & Lamiaceae & Herb & Root [24] \\
\hline 98 & Senna alexandrina mill & Senna & Fabaceae & Herb & Leaf, Fruit (Fruit, Seed) \\
\hline 99 & Sesamun indicum & Sesame & Pedaliaceae & Tree & Leaf and Wood \\
\hline 100 & Silybum marianum & Cardus marianus & Asteraceae & Herb & Fruit (Seed) \\
\hline 101 & Smyrnium cordifolium & Smyrnium & Apiaceae & Herb & Seed \\
\hline 102 & Strychnos nux-vomica & Vishatinaduka & Loganiaceae & Tree & Fruit (Seed), Stem Or Bark \\
\hline 103 & Suaeda aegyptiaca & Suaeda & Amaranthaceae & Herb & Leaf \\
\hline 104 & Symplocos racemosa Roxb. & Symplocos & Symplocaceae & Shrub & Bark [25] \\
\hline 105 & Tamarindus indica $\mathrm{L}$. & Tamarind & Fabaceae & Tree & Leaf and fruit \\
\hline 106 & Taraxacum officinale & Dandelion & Asteraceae & Herb & Root (Rhizome) \\
\hline 107 & Taxus baccata & Yew & Taxaceae & Shrub & Leaf \\
\hline 108 & Terminalia arjuna (roxb.) & Arjuna & Combretaceae & Tree & Fruit (Seed), Bark (Stem) \\
\hline 109 & Terminalia chebula Willd. ex & Myrobalan & Combretaceae & Tree & Fruits \\
\hline 110 & Terminalia horrida Stoud. & Terminalia & Santalaceae & Tree & Fruits \\
\hline 111 & Thymus serpyllum L. & Breckland thyme & Lamiaceae & Herb & Areal part \\
\hline 112 & Trachyspermum ammi & Ajwain & Apiaceae & Herb & Fruit \\
\hline 113 & Tragopogon porrifolius & Yellow salsify & Asteraceae & Herb & Root [26] \\
\hline 114 & Trigonella foenum-graecum & Fenugreek & Fabaceae & Herb & Fruit (Seed) \\
\hline 115 & Ulmus glabra Hudson. & Scotch elm & Ulmaceae & Tree & Leaf \\
\hline 116 & Urtica dioica & Nettle & Urticaceae & Herb & Leaf and branches \\
\hline 117 & Usnea barbata Ach. & Usnea barbata & Parmeliaceae & Herb & Filaments \\
\hline 118 & Valeriana officinalis & Valerian & Caprifoliaceae & Herb & Fruit \\
\hline 120 & Vitis vinifera & Grape vine & Vitaceae & Shrub & Fruit \\
\hline 121 & Withania somnifera & Ashwagandha & Solanaceae & Herb & Root, Whole Plant \\
\hline 122 & Zingiber officinale & Sondh & Zingiberaceae & Herb & Root, Whole Plant \\
\hline 123 & Zingiber officinale roscoe & Ginger & Zingiberaceae & Herb & Root, Whole Plant \\
\hline 124 & Zingiber zerumbet (L.) Sm. & Lempoyang & Zingiberaceae & Herb & $\begin{array}{l}\text { Pseudo-stem (https://en.wikipedia.org/wiki/ } \\
\text { Scutellaria\#traditional_use) }\end{array}$ \\
\hline 125 & Ziziphus jujuba(l) h.karst & Indian date & Rhamnaceae & Tree & Fruit \\
\hline 127 & Ziziphus nummularia & Jhar ber & Rhamnaceae & Shrub & Flower, leaf and Fruit \\
\hline 128 & Ziziphus spina-christi & Thorn jujube & Rhamnaceae & Tree & Leaf and Stem \\
\hline
\end{tabular}




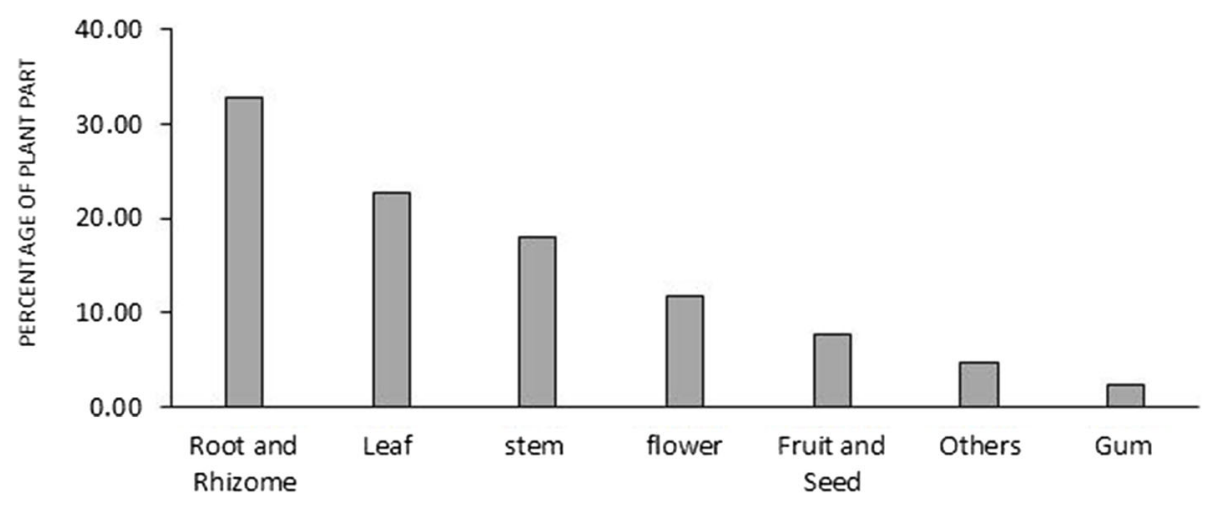

Fig. 1 The percentage of plant parts used for the treatment of Heart Disease

herbal medicine though exist since past decade, this system of medicine has several lacunae. For example, herbal medicine lack scientific evidence or assessment. Many of the herbal medicines have toxic effects and major drugdrug interactions too. Therefore, in-depth research is needed to understand the pharmacological activity of the herbs. In this paper, cluster analysis of the potential herbs for heart with its ability to treat various heart related diseases was done to understand the possible combination of the herbs that may help in the development of more effective drug formulation that the existing one. As mentioned in the results section, almost every part of the herb has a medicinal property, although root and rhizome is most frequently used plant parts.

Herbs have more than one medicinal property i.e. it has the potential to prevent or cure more than one disease as demonstrated in our results. For example, only one herb Crocus sativus L. was found to have medicinal property with the potential to treat five heart disease type-hypertension, heart attack, reduction in blood fat, anti- oxidant, and cardiac tonic. Role of this herb against cardiovascular diseases is related to their antioxidant and anti-inflammation effects [31]. Crocus sativus is found to have antihypertensive and normalizing effect on blood pressure [32]. It is known to possess a potent inhibitory effect on heart rate and contractility of guinea pig heart via calcium channel-blocking effect [33]. Other studies also support cardiovascular effects of saffron and its components [34].

Three herbs-Citrus medica L., Crataegus monogyna, Elettaria cardamom possess medicinal property with the potential to treat four heart disease type. Citrus medica L. "Otroj" (Brain citron), is a member of Rutaceae family. Evidence supports its cardioprotective potential due to its potent antioxidant and free radical scavenging activity [35]. Crataegus species is shown to represent a safe, effective, nontoxic agent in the treatment of cardiovascular disease and ischemic heart disease (IHD) [36]. Its mechanism of action include direct scavenging of reactive oxygen species, enhanced superoxide dismutase, and catalase activities, antioxidant activity, down regulation of caspase 3 gene etc. [36]. Crataegus monogyna are rich in polyphenols and both of its leaves and flowers or alternatively the fruit are used medicinally [37]. It helps to regulate both high and low blood pressure, in addition to slowly breaking down cholesterol and fat deposits in the body [37]. It increases conversion rates of LDL or "bad" cholesterol into HDL or "good" cholesterol in the liver and improves blood and oxygen supply to the heart muscle. In cases of congestive heart failure and circulatory disorders, Hawthorns is prominently being used in a holistic approach to heal the body itself [37]. It plays a role in alleviating irritation and swelling of the blood vessels. Study has shown that small cardamom Elettaria cardamom effectively lowers blood pressure, increases fibrinolysis, and boosts antioxidant status in stage 1 hypertensive patients without affecting blood lipids or fibrinogen levels [38]. A rat study has also demonstrated the ability of cardamom oil to restore lipid homeostasis in the presence of hypercholesterolemia [39]. This study has shown reduction in atherogenicity index by dietary intervention with cardamom powder and cardamom oil hence, the cardioprotective potential of cardamom [39]. The bark of Terminalia arjuna has been demonstrated to show cardioprotective effects against doxorubicin induced cardiotoxicity by increased coronary artery flow and protection of myocardium against ischemic damage [40]. Terminalia chebula pericap has also been reported to have cardioprotective activity [41].

The dendogram obtained for 128 medicinal plants by Jaccard Neighbour joining dendogram method depicts 128 different medicinal plants having property to cure some type of heart disease/ailment. The medicinal plants in one group showed to have similar properties to cure same heart disease. The distance in dendogram depicts closeness of the properties curing the heart disease; less the distance between two medicinal plants or two groups, the more it is closer to cure particular 
Table 2: Heart disease and the Plant Uses (A-C)

\section{A. Plants used in only one disease of heart}

Reduction of blood pressure

Angina

Anti-oxidant

Treatment of blocked arteries

Cardio myopathies

Blood purification

Congenital heart disease

Heart attack

Hypertension

Reduction in blood fat

Nourishing of the heart

Increase in venous blood

Cardiac tonic

Cardiac disorders and arrhythmias

\section{B. Plants used in two diseases of heart}

Hypertension, heart attack

Reduction of blood pressure, reduction in blood fat

Anti-oxidant, cardiac tonic

Angina, hypertension

Reduction of blood pressure, blood purification

Reduction in blood fat, anti-oxidant

Blood purification, reduction in blood fat

Angina, anti-oxidant

Angina, heart attack

Blood purification, reduction in blood fat

Reduction in blood fat, anti-oxidant

Reduction of blood pressure, treatment of hypolipidemia

Reduction of blood pressure, anti-oxidant

Hypertension, cardiac tonic

Hypertension, anti-oxidant

Hypertension, reduction in blood fat

Angina, reduction in blood fat

\section{Plants used in three diseases of heart}

Angina, hyper-tension, heart attack

Hypertension, anti-oxidant, cardiac tonic

Hypertension, reduction in blood fat, cardiac tonic

Congenital heart disease, reduction in blood fat, cardiac tonic

Reduction of blood pressure, blood purification, reduction in blood fat

Angina, reduction in blood fat, anti- oxidant
Achillea millefolium, Ecbalium elaterium, Falcaria vulgaris, Prunus scoparia (spach) Schneider, Berberis integrima, Crocus haussknechtii, Olea europaea, Silybum marianum, Smyrnium cordifolium, Taxus baccata, Tragapogon caricifolius, Ziziphus nummularia

Actaea racemosa, Camellia sinensis, Garcinia cambogia (gaertn.) Desr., Genus panax, Ginkgo biloba, Medicago sativa, Ruscus aculeatus

Amomum subulatum Roxb, Anchusa italica Retz., Bambusa arundinacea Retz., Boerhavia diffusa, Boswellia carteri Birdw., Calamintha acinos (L.) Clairv., Centaurea behen L., Cinnamomum cassia (L.), Curcuma zedoaria (Bergius), Lavandula stoechas L., Pistacia vera L., Pterocarpus santalinus L.f., Rosax damascena Mill., Symplocos racemosa Roxb., Tamarindus indica L., Terminalia horrida Stoud., Usnea barbata Ach., Zingiber zerumbet (L.) Sm.

Anthemis gayana Boiss.

Apium graveolens, Strychnos nux-vomica

Arctium minus hill., Juniperus communis, Malva neglecta, Portulaca oleracea, Rhus coriaria, Rosa canina, Rubus caesius, Suaeda aegyptiaca

Carissa carandas linn., Garcina indica choisy, Garcinia pedunculata roxb., Gmelina asiatica linn., Magnifera indica linn. Carthamus tinctorius, Inula helenium

Centella asiatica (I.) Urban, Cinnamomum verum, Crataegus pontica c. Koch., Myristica fragrans, Nigella sativa, Paeonia officinalis L., Paliurus spina-christi miller., Piper longum, Piper nigrum, Scutellaria pekinensis, Terminalia arjuna (roxb.), Withania somnifera, Zingiber officinale

Coriandrum sativum, Gundelia tournefortii, Lactuca sativa, Mentha spicata, Polypodium vulgare L., Senna alexandrina mill, Sesamun indicum, Trigonella foenum-graecum, Urtica dioica

Fragaria vesca $L$.

Melilotus indicus

Nerium oleander $L$.

Ulmus glabra Hudson.

Acorus calamus, Aloe vera, Commiphora myrrha, Leonurus cardiac, Picrorrhiza kurroa

Anethum graveolens

Aquilaria agallocha Roxb.

Berberis darwinii, Capsicum annuum, Valeriana officinalis

Berberis vulgaris, Ziziphus spina-christi

Calendula officinalis L., Melissa officinalis L., Melissa officinalis L., Mentha $\times$ piperita L.

Cichorium intybus

Cinnamomum camphora L.

Commiphora wightii

Eclipta prostrata

Malus domestica Baumg., Trachyspermum ammi

Nectaro scordeum tripedale

Nymphaea alba L.

Phyllanthus emblica $L$.

Pyrus communis L., Rheum ribes, Thymus serpyllum L.

Santalum album L., Vitis vinifera

Zingiber officinale roscoe

Allium sativum, Curcuma longa

Nardostachys jatamansi

Ocimum bacilicum

Punica granatum linn

Rumex crispus

Taraxacum officinale 
Table 2: Heart disease and the Plant Uses (A-C) (Continued)

\begin{tabular}{ll}
\hline Reduction in blood fat, anti-oxidant, cardiac tonic & Terminalia chebula Willd. ex \\
$\begin{array}{l}\text { Cardio myopathies, congenital heart disease, } \\
\text { reduction of blood pressure }\end{array}$ & Ziziphus jujuba(l) h.karst
\end{tabular}

\title{
D. Plants used in four diseases of heart
}

\begin{abstract}
Congenital heart disease, reduction in blood fat, Citrus medica $\mathrm{L}$. anti- oxidant, cardiac tonic

Angina, hypertension, heart attack, nourishing of Crataegus monogyna, the heart,

Hypertension, cardio myopathies, reduction of Elettaria cardamom blood pressure, anti-oxidant
\end{abstract}

\section{E. Plants used in five diseases of heart}

Hypertension, heart attack, reduction in blood fat, Crocus sativus $L$. anti- oxidant, cardiac tonic

(Source: Babak Baharvand-Ahmadi Pathophysi et. al., 2017 [27])

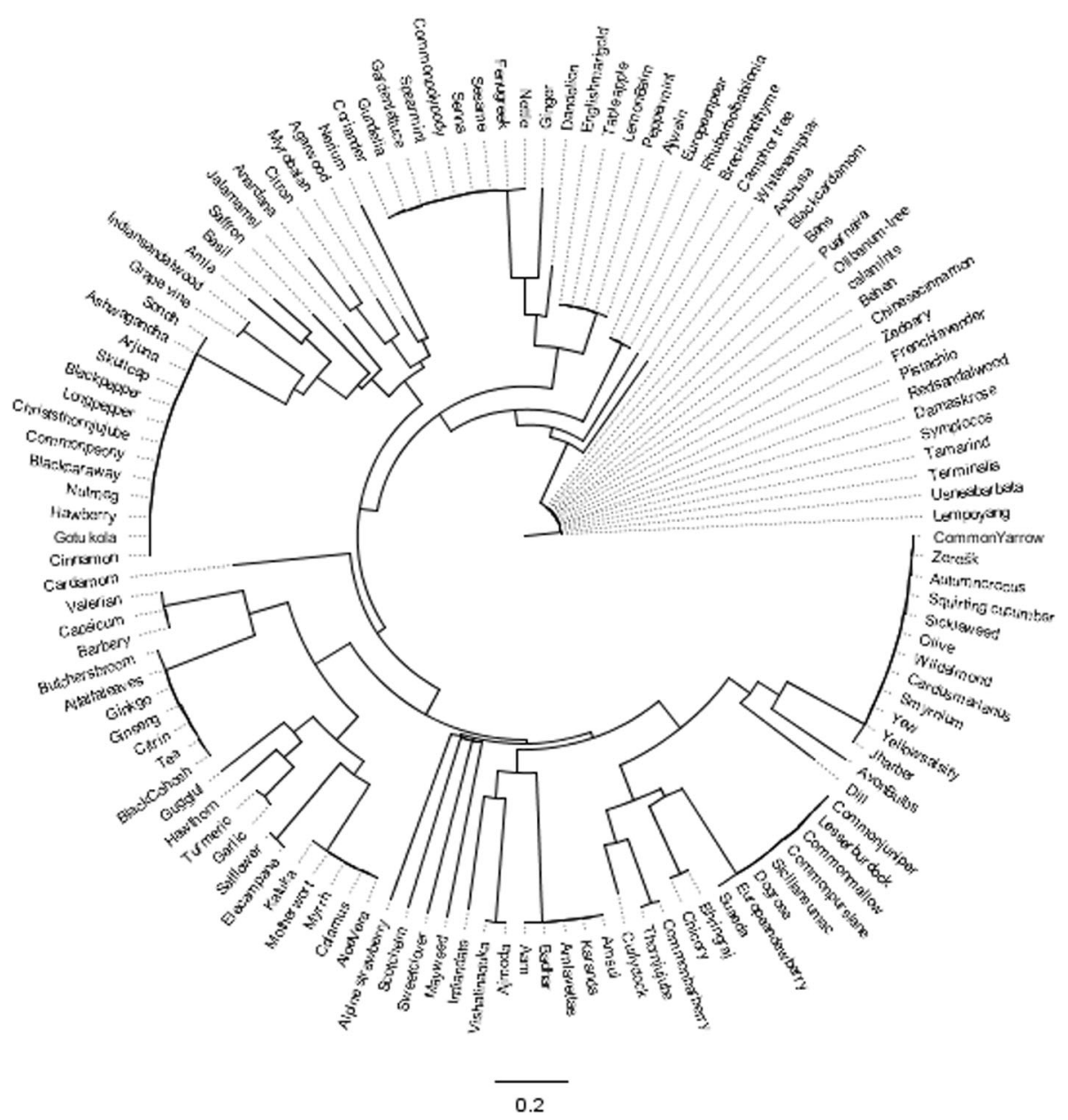

Fig. 2 The dendogram depicts 128 different medicinal plants having property to cure some type of heart disease/ailment. The medicinal plants in one group shows that they have similar properties to cure same heart disease. The distance in dendogram depicts closeness of properties curing heart disease; as less the distance between two medicinal plants or two groups they will more close to cure particular heart disease 
heart disease. During drug development, a medicinal plant can be replaced by another plant of same group or by another plant of its neighbour group but from same pedigree (like garlic, turmeric and safflower) on the basis of their similar properties of curing that particular heart disease. Thus, in case of non-availability of the herbs or if it belongs to the category of rare, threatened, and endangered species, such method may add to new ways of drug development. During drug development, if any medicinal plant shows adverse effect, it can be replaced by a plant with similar medicinal potential, suitable for the same drug composition based on cluster analysis.

Generally, herbal medicines is considered harmless as it is derived from natural sources, however, adverse reaction of herbal medicines is also reported. For example, bleeding is the adverse effect of the herbal drug, Ginko biloba. Similarly, gastrointestinal disturbances, allergic reactions, fatigue, dizziness, confusion, dry mouth, photosensitivity are the adverse effect of the herbal drug St. John's wort. Lack of information on the social and economic benefits on the industrial utilization of medicinal plants is the major drawback in development of the medicinal plant-based industries in developing countries $[7,8]$.

Medicinal plants are the oldest known health-care products and its importance in the primary health care of individuals and communities in both developed as well as developing countries is increasing. However, further research is needed to find compounds of interest in these plants that can be used as safe and effective medicines to treat heart disease.

\section{Conclusion}

More scientific research on these plants is needed in order to find new drugs for the treatment of cardiovascular diseases that have no or few side effects.

\section{Acknowledgements}

Authors sincerely thank Director, CSIR-NISTADS (Pusa, New Delhi) for providing constant support and encouragement in completing this manuscript.

\section{Authors' contributions}

Suman Ray has provided the concept of the manuscript and written the draft of the manuscript. Tables, Figures and analysis was contributed by Mahesh Kumar Saini. Both authors read and approved the final manuscript.

\section{Funding}

Funding support from NISTADS/OLP/2017/2.

\section{Availability of data and materials}

Not applicable

\section{Declarations}

Ethics approval and consent to participate Not applicable
Consent for publication

Prior consent of all the authors is taken.

\section{Competing interests}

The authors declare that they have no competing interests.

Received: 27 January 2021 Accepted: 10 June 2021

Published online: 14 July 2021

\section{References}

1. Wu BN, Huang YC, Wu HM, Hong SJ, Chiang LC, Chen J. A highly selective $\beta 1$-adrenergic blocker with partial $\beta 2$-agonist activity derived from ferulic acid, an active component of Ligusticum wallichii Franch. J Cardiovasc Pharmacol. 1998;31(5):750-7.

2. World Health Organization. Burden: mortality, morbidity and risk factors. In: Alwan A, editor. Global status report on non-communicable diseases 2010. Geneva: World Health Organization; 2011.

3. Trikramji AJ, editor. Charak Samhita. 1st Adhyaya, 5th ed. Varanasi: Chaukhambha Sanskrit Sansthan; 2001;23.

4. Malik V. Lucknow: Eastern Book Company;2007. Laws relating to drugs and cosmetics. 1940;52-3.

5. Saper RB, Kales SN, Paquin J, Burns MJ, Eisenberg DM, Davis RB, et al. Heavy metal content of ayurvedic herbal medicine products. JAMA. 2004;292(23): 2868-73.

6. Parab S, Kulkarni R, Thatte U. Heavy metals in 'herbal' medicines. Indian J Gastroenterol. 2003;22(3):111.

7. Dahanukar SA, Thatte UM. Can we prescribe ayurvedic drugs rationally? Indian Pract. 1998;51:882-6.

8. Sharma PK. Pharmacovigilance: a need in ayurvedic medicine system. Int J Green Pharm. 2018;12:01.

9. Kubde S. Adverse drug reactions and pharmacovigilance of herbal medicines in India. Int J Green Pharm. 2016;10(1):S29-32.

10. Liperoti R, Vetrano DL, Bernabei R, Onder G. Herbal medications in cardiovascular medicine. J Am Coll. 2017;69(9):1188-99.

11. Baharvand-Ahmadi B, Bahmani M, Eftekhari Z, Jelodari M, Mirhoseini M. Overview of medicinal plants used for cardiovascular system disorders and diseases in ethnobotany of different areas in Iran. J Herb Med Pharmacol. 2015:5(1):39-44.

12. Wuttke W, Seidlova-Wuttke D, Gorkow C. The cimicifuga preparation BNO 1055 vs. conjugated estrogens in a double-blind placebo-controlled study: effects on menopause symptoms and bone markers. Maturitas. 2003;44: S67-77.

13. Mosaddegh M, Esmaeili S, Eslami-Tehrani B, Kermatian B, Mohebby S, Hamzeloo-Moghadam M. Anti-angiogenesis properties of Crocus pallasii subsp. haussknechtii, a popular ethnic food. Res J Pharmacogn. 2015;2(3):3742.

14. Yob NJ, Jofrry SM, Affandi MM, Teh LK, Salleh MZ, Zakaria ZA. Zingiber zerumbet (L.) Smith: a review of its ethnomedicinal, chemical, and pharmacological uses. Evid Based Complement Altern Med. 2011;543216.

15. Baharvand-Ahmadi B, Bahmani M, Tajeddini P, Rafieian-Kopaei M, Naghdi N. An ethnobotanical study of medicinal plants administered for the treatment of hypertension. J Renal Injury Prev. 2016;5(3):123.

16. Nagore DH, Bhusnar HU, Nipanikar SU. Phytopharmacological profile of Symplocos racemosa: a review. Pharmacologia. 2014;5(2):76-83.

17. Rasha HM, Salha A, Thanai A, Zahar A. The biological importance of Garcinia Cambogia: a review. J Nutr Food Sci. 2015;55:004.

18. Coon JT, Ernst E. Panax ginseng. Drug Saf. 2002;25(5):323-44.

19. Biloba G. Woodland Publishing. Pleasant Grove, UT. 1996.

20. Russell RG, Rogers MJ, Frith JC, Luckman SP, Coxon FP, Benford HL, et al The pharmacology of bisphosphonates and new insights into their mechanisms of action. J Bone Miner Res. 1999;14(S2):53-65.

21. Keller K. Assessment report on Leonurus cardiaca L., herba. KELLER, K. Committee on Herbal Medicinal Products (HMPC). 2010.

22. Baharvand-Ahmadi B, Bahmani M, Tajeddini P, Naghdi N, Rafieian-Kopaei M. An ethno-medicinal study of medicinal plants used for the treatment of diabetes. J Nephropathol. 2016:5(1):44.

23. Ghaseminasab M, Ahmadi A, Mazloomi SM. A review on pistachio: its composition and benefits regarding the prevention or treatment of diseases. JOHE. 2015;4(1):57-69. 
24. Sorkheh K, Kiani S, Sofo A. Wild almond (Prunus scoparia L.) as potential oilseed resource for the future: Studies on the variability of its oil content and composition. Food Chem. 2016;212:58-64.

25. Kaur R, Arya V. Ethnomedicinal and phytochemical perspectives of Pyrus communis Linn. J Pharmacogn Phytochem. 2012;1(2):14-9.

26. Redman DA. Ruscus aculeatus (butcher's broom) as a potential treatment for orthostatic hypotension, with a case report. J Altern Complement Med. 2000;6(6):539-49.

27. Baharvand-Ahmadi B, Asadi-Samani M. A mini-review on the most important effective medicinal plants to treat hypertension in ethnobotanical evidence of Iran. J Nephropharmacol. 2017;6(1):3.

28. Patidar SK, Mitra M, George B, Soundarya R, Mishra S. Potential of Monoraphidium minutum for carbon sequestration and lipid production in response to varying growth mode. Bioresour Technol. 2014;172:32-40.

29. Balick MJ, Cox PA. Plants, people, and culture: the science of ethnobotany. Scientific American Library; 1996.

30. Mashour M. Qadaya asasiya fi-tariq al-da'wa. Cairo: Dar al-Tawzi'wa-la-Nashr al-Islamiyya. 1988

31. Sargolzaei J, Shabestari MM. The effects of Crocus Sativus L. and its main constituents against cardiovascular diseases. Der Pharmacia Lettre. 2016; 8(13):38-41.

32. Imenshahidi M, Razavi BM, Faal A, Gholampoor A, Mousavi SM, Hosseinzadeh $\mathrm{H}$. The effect of chronic administration of saffron (Crocus sativus) stigma aqueous extract on systolic blood pressure in rats. Jundishapur J. 2013;8(4):175.

33. Boskabady MH, Shafei MN, Shakiba A, Sefidi HS. Effect of aqueous-ethano extract from Crocus sativus (saffron) on guinea-pig isolated heart. Phytother Res. 2008:22(3):330-4.

34. Mehdizadeh R, Parizadeh MR, Khooei AR, Mehri S, Hosseinzadeh H. Cardioprotective effect of saffron extract and safranal in isoproterenolinduced myocardial infarction in wistar rats. Iran J Basic Med Sci. 2013;16(1): 56.

35. Al-Yahya MA, Mothana RA, Al-Said MS, El-Tahir KE, Al-Sohaibani M, Rafatullah S. Citrus medica "Otroj": attenuates oxidative stress and cardiac dysrhythmia in isoproterenol-induced cardiomyopathy in rats. Nutrients. 2013;5(11):4269-83.

36. Tassell MC, Kingston R, Gilroy D, Lehane M, Furey A. Hawthorn (Crataegus spp.) in the treatment of cardiovascular disease. Pharmacogn Rev. 2010;4(7): 32

37. Altinterim B. Cardio vascular effects of Hawthorn (Crataegus monogyna). KSÜ DOĞA BILIMLERI DERGISI. 2012;15(3):16-18.

38. Verma SK, Jain V, Katewa SS. Blood pressure lowering, fibrinolysis enhancing and antioxidant activities of cardamom (Elettaria cardamomum). Indian J Biochem Biophys. 2009:46(6):503-6.

39. Nagashree S, Archana KK, Srinivas P, Srinivasan K, Sowbhagya HB. Antihypercholesterolemic influence of the spice cardamom (Elettaria cardamomum) in experimental rats. J Sci Food Agric. 2017;97(10):3204-10.

40. Singh G, Singh AT, Abraham A, Bhat B, Mukherjee A, Verma R, et al. Protective effects of Terminalia arjuna against Doxorubicin-induced cardiotoxicity. J Ethnopharmacol. 2008:117(1):123-9.

41. Reddy VR, Kumari SR, Reddy BM, Azeem MA, Prabhakar MC, Rao AA. Cardiotonic activity of the fruits of Terminalia chebula. Fitoterapia. 1990; 41(6):517-25.

\section{Publisher's Note}

Springer Nature remains neutral with regard to jurisdictional claims in published maps and institutional affiliations.

\section{Submit your manuscript to a SpringerOpen ${ }^{\circ}$ journal and benefit from:}

- Convenient online submission

- Rigorous peer review

- Open access: articles freely available online

- High visibility within the field

- Retaining the copyright to your article

Submit your next manuscript at $\boldsymbol{\nabla}$ springeropen.com 